\title{
Reinforcement of concrete by composite materials
}

\section{Renforcement du béton par les matériaux composites}

\author{
Bensaid Boulekbache, Mostefa Hamrat, Nejma Imane Gacem, Kheltoum Halimi \\ Université Hassiba Benbouali de Chlef, Algérie
}

\begin{abstract}
This work was devoted to the material-scale study of the mechanical behavior of confined concrete by composite materials based on carbon fiber and glass under compression and bending loading. In this context, an experimental work on cylindrical and prismatic concrete specimens has been carried out. The obtained results permitted the identification of the mechanical behavior, strength, deformability and failure modes. The outcome of the present research indicates an increase in the bearing capacity and deformability in compression and in tension. The reinforcement by means of composite materials appears as a pertinent solution and therefore, makes it possible to obtain clear indication on the type of confinement to be considered according to the intended purpose.
\end{abstract}

\begin{abstract}
Résumé. Ce travail a été consacré à l'étude à l'échelle du matériau du comportement mécanique du béton confiné par des matériaux composites à base de fibres de carbone et de verre sous un chargement de compression et flexion. Dans ce sens, un travail expérimental sur des spécimens en béton de forme cylindrique et prismatique a été réalisé. Les résultats expérimentaux ont permis l'identification comportement mécanique, des résistances mécaniques et la déformabilité et les modes de rupture. Pour l'ensemble des résultats obtenus, on a pu constater un accroissement de la capacité portante et de la déformabilité rn compression et en traction, Le renforcement par les matériaux composite est une solution fiable pour le renforcement et permet de dégager des indications claires sur le confinement à adopter selon l'objectif visé.
\end{abstract}

\section{Introduction}

Le béton est un matériau qui se modifie tout au long de sa vie utile, car la structure dont il fait partie subit ellemême des changements. Ces changements proviennent soit de modifications ou de rénovations, soit d'une altération de sa capacité de support par un accroissement des charges. Dans la plupart des cas, ça nécessite un renfort.

Le renforcement d'une structure en béton consiste à améliorer les caractéristiques mécaniques des éléments qui la composent, de manière à ce qu'elle offre une meilleure solidité aussi bien en état de service qu'en état de résistances ultimes. Il s'agit principalement des techniques de renfort avec une incorporation de profiles métalliques, ou bien une modification de la section structurelle et la plus récente qui utilise des matériaux composites en tant que renfort externe.
Si une structure est bien conçue et correctement exécutée, elle n'aura besoin d'aucun renforcement au moment de sa mise en service. Cependant, suite à des erreurs dans le projet ou dans l'exécution, ou suite à diverses circonstances lors de la vie en service (chargements maximums dépassés, changement des conditions d'exploitation, fluage, fatigue, attaques chimiques dues à l'environnement...), des renforts peuvent s'avérer nécessaires. Ce changement de circonstances implique que la nouvelle structure, issue du « projet actualisé », soit plus exigeante que l'antérieure.

Ainsi la notion de réparation vise à restituer les caractéristiques initiales de portance (rigidité-résistance). Le renforcement a pour objectif d'améliorer les performances de la structure vis-à-vis de conditions d'exploitations modifiées ou d'augmenter la durée de vie de l'ouvrage. Le principal avantage du renforcement par 
composites est la manipulation très facile sur chantier sans recours au matériel lourd.

\section{Programme expérimental}

\subsection{Matériaux et matériel utilisés}

Les bétons sont confectionnés dans un malaxeur d'une capacité de 50 litres. La formulation du béton testé est donnée dans le tableau 1.

Tableau 1. Composition massique en $\mathrm{kg} / \mathrm{m}^{3}$ du béton.

\begin{tabular}{|c|c|c|c|c|}
\hline Ciment & Eau & Sable (0/4) & Gravier 8/15 & E/C \\
\hline 350 & 210 & 680 & 1135 & 0,6 \\
\hline
\end{tabular}

Dans les essais réalisés pour l'étude des bétons, deux types d'éprouvettes ont été utilisés:

- Eprouvettes cylindrique de dimensions $10 \times 20 \mathrm{~cm}$, pour les essais de compression.

- Eprouvettes prismatiques de dimension $7 \times 7 \times 28 \mathrm{~cm}$ pour les essais de flexion.

Les écrasements ont été effectués à 28 jours d'âge.

\subsection{Matériaux composites}

Les matériaux composites utilisés dans cette étude est composé d'une résine époxy et des tissus en fibre de carbone et en fibre de verre (Figures 1 et 2 ).

-PRFC : Polymère renforcé de fibre de carbone

-PRFG : Polymère renforcé de fibre de verre

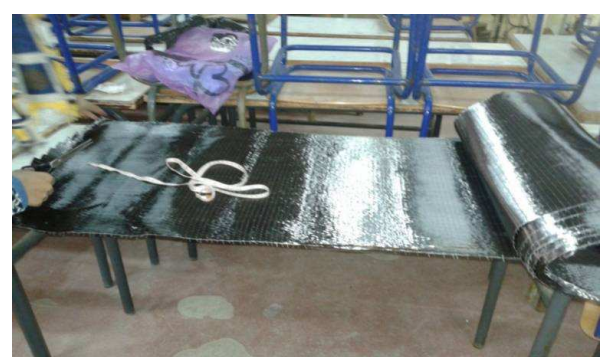

Fig. 1. Tissu de fibres de carbone

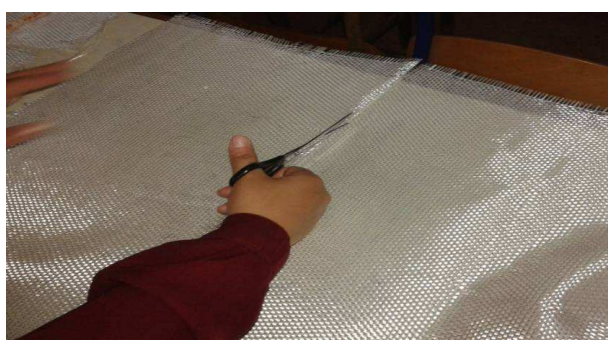

Fig 2. Tissu de fibres de verre

Les propriétés mécaniques fournies par le fabricant sont données dans le Tableau 2.

Tableau 2. Propriétés du PRFC \& PRFG et de SIKADUR

\begin{tabular}{|c|c|c|c|}
\hline & SikaWrap & Verre & Sikadur 330 \\
\hline $\begin{array}{c}\text { Résistance à la } \\
\text { traction }\end{array}$ & $4300 \mathrm{MPa}$ & $2,2 \mathrm{GPa}$ & $30 \mathrm{MPa}$ \\
\hline $\begin{array}{c}\text { Allongement à la } \\
\text { rupture }\end{array}$ & $1,8 \%$ & $4,8 \%$ & 2 à $5 \%$ \\
\hline
\end{tabular}

\begin{tabular}{|c|c|c|c|}
\hline $\begin{array}{c}\text { Module d'élasticité } \\
\text { en traction }\end{array}$ & $234 \mathrm{GPa}$ & $72 \mathrm{GPa}$ & $4,5 \mathrm{GPa}$ \\
\hline Epaisseur & $0,13 \mathrm{~mm}$ & $1,1 \mathrm{~mm}$ & $/$ \\
\hline Densité & 1,8 & 2,54 & $1,3 \mathrm{~kg} / \mathrm{l}$ \\
\hline
\end{tabular}

\subsection{Préparation des surfaces du béton}

Le support doit être propre, sec, exempt de toute trace de graisse, d'huile, de rouille, de produit de cure, d'ancien revêtement ou traitement de surface, de parties non ou peu adhérentes, de laitance.

Avant l'utilisation des fibres (carbone ou verre), on a effectué un ponçage sur l'ensemble des éprouvettes afin d'éliminer la laitance de ciment, les particules friables non ou peu adhérents, et d'ouvrir la porosité de surface. Avant l'application de la résine Sikadur-330, toutes les poussières et autres particules non ou peu adhérentes doivent être complètement éliminées du support de préférence par brossage (papier verre 80) puis par aspiration mécanique (Figure 3).
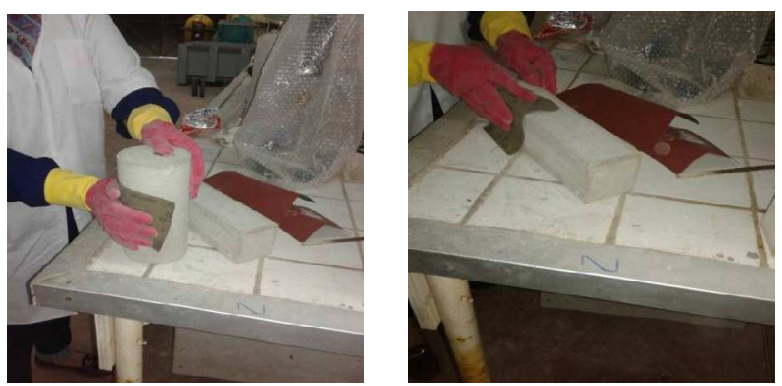

Fig 3. Ponçage des éprouvettes

\subsection{Préparation de la résine époxy Sikadur-330}

La résine à base d'époxy à deux composantes, résine (A) et durcisseur (B), doit être mélangée peu de temps avant l'application.

La proportion en masse du durcisseur représentait $25 \%$ de la masse de la résine (durcisseur/résine $=0,25$ ) conformément aux recommandations du fournisseur. Le malaxage a été réalisé à faible vitesse, afin d'éviter l'inclusion d'air, pendant trois minutes environ jusqu'à disparition complète des traînées de couleur et l'obtention d'un mélange homogène. Le temps d'application de la résine (durée de vie en pot) était d'environ 60 minutes à $10^{\circ} \mathrm{C}$ ou 30 minutes à $35^{\circ} \mathrm{C}$. La durée de vie en pot démarre dès le malaxage des deux composants (résine et durcisseur). Après cette période de temps, la résine est inutilisable. A basse température cette durée de vie sera plus longue qu'à haute température. Plus la quantité de mélange est importante, plus cette durée est courte. Afin d'augmenter cette durée lors de haute température, il est conseillé de répartir le mélange dans plusieurs récipients ou de refroidir chaque composant avant d'effectuer le mélange. Un mûrissement d'environ 5 jours à $23^{\circ} \mathrm{C}$ est requis pour la cure de la résine, selon les recommandations du fabricant. Ces temps indicatifs peuvent varier suivant les conditions ambiantes particulières à chaque application. Tous les spécimens ont bénéficié d'un temps de cure supérieur ou égale aux 
recommandations du fabricant (une semaine pour les spécimens).

\subsection{Mise en œuvre de PRF}

Le renforcement de structures à partir d'un tissu sec se fait directement par la pose de ce dernier sur une couche de résine qui recouvre l'élément à renforcer et qui sert de liant. Après marouflage, une couche de résine de fermeture permet de parfaire l'imprégnation. Les pressions nécessaires appliquées pour cette méthode de renforcement sont faibles par rapport à d'autres méthodes de renforcement. La direction principale des fibres a été orientée dans le sens circonférentiel des cylindres et longitudinal des prismes.

Cette technique permet notamment un suivi parfait de la forme du support ainsi que la maîtrise de l'épaisseur du film de résine. En utilisant des polymères qui polymérisent à température ambiante, il est possible de réaliser une stratification directe, en respectant les étapes suivantes :

- préparation de la surface, application de la couche primaire,

- application d'une première couche de polymère époxyde. La colle est constituée de 2 composants (résine + durcisseur),

- application d'une première couche de tissus secs,

- application d'une seconde couche de polymère époxyde, protection, finition

Ce procédé présente l'avantage d'une mise en œuvre simplifiée, mais le renforcement acquiert toute sa résistance au bout d'une semaine et le contrôle de la qualité de l'application doit être soigné suivant les conditions de mise en œuvre.

\section{Résultats et discussions}

\subsection{Essai de compression simple}

Les résultats des essais de laboratoire sont présentés numériquement dans le tableau.

Le Tableau 3 présente les valeurs maximales obtenues des résistances en compression, des déformations axiales, des augmentations en résistance à la compression des cylindres confinés avec PRFG et PRFC et les allongements à la rupture par rapport au béton témoin.

Tableau 3. Résultats des essais sur les cylindres en béton.

\begin{tabular}{|c|c|c|c|}
\hline $\begin{array}{c}\text { Nombre } \\
\text { de } \\
\text { couches }\end{array}$ & $\begin{array}{c}\text { Résistance à la } \\
\text { compression } \\
(\mathrm{MPa})\end{array}$ & $\begin{array}{c}\text { Augmentations } \\
\text { des résistances } \\
(\%)\end{array}$ & $\begin{array}{c}\text { Allongement } \\
\text { A la rupture } \\
(\%)\end{array}$ \\
\hline $\mathrm{T}$ & 20 & - & 2,1 \\
\hline $\mathrm{V} 1$ & 26 & 63 & 11,65 \\
\hline $\mathrm{V} 2$ & 35 & 120 & 18,45 \\
\hline $\mathrm{V} 3$ & 45 & 180 & 19.6 \\
\hline $\mathrm{C} 1$ & 56 & 260 & 24,8 \\
\hline $\mathrm{C} 2$ & 88 & 450 & $29, .92$ \\
\hline $\mathrm{C} 3$ & 113 & 607 & 35 \\
\hline
\end{tabular}

$\mathrm{T}$ : éprouvette témoin

V1 : éprouvette renforcée par 1 couche de fibre de verre
V2 : éprouvette renforcée par 2 couches de fibre de verre V3 : éprouvette renforcée par 3 couches de fibre de verre $\mathrm{C} 1$ : éprouvette renforcée par 1 couche de fibre de carbone $\mathrm{C} 2$ : éprouvette renforcée par 2 couches de fibre de carbone C3 : éprouvette renforcée par 3 couches de fibre de carbone

Le confinement de 1, 2 et 3 couches de PRFG, en comparaison avec les spécimens non confinés conduit respectivement à une augmentation de la résistance à la compression de $63 \%, 120$ et $180 \%$ ainsi que les déformations à la rupture.

Le confinement de 1, 2 et 3 couches de PRFC, en comparaison avec les spécimens non confinés conduit respectivement à une augmentation de la résistance à la compression des spécimens cylindriques de $260 \%, 450 \%$ et $607 \%$ ainsi que les déformation à la rupture.

Ces résultats montrent que le confinement améliore considérablement la résistance et la capacité de déformation du béton confiné.

Un cylindre (poteau) soumis à la compression, renforcé par confinement aura l'avantage d'augmenter sa capacité en résistance en même temps que sa déformation. Le confinement circonférentielle des colonnes donne les meilleures performances en matière de résistance à la compression et déformation axiale.

La majorité des éprouvettes en béton confiné avec des matériaux composites PRF ont présenté une allure caractéristique bilinéaire ascendante (Figure 4). Dans la première branche linéaire, l'inclinaison de la courbe contrainte-déformation est semblable à celle du béton ordinaire, ce qui montre que le comportement est peu affecté par l'enveloppe composite. Dans la seconde branche linéaire, le béton est fissuré et la force de confinement du PRF est activée. La contrainte du béton confiné augmente linéairement avec l'augmentation de la contrainte dans le composite et atteint son maximum à la rupture de l'enveloppe PRF.

L'enveloppe en matériaux composites exerce une pression latérale sur le béton pour contrecarrer le gonflement créé par la dégradation de la rigidité du béton. La troisième branche prend naissance lorsque l'enveloppe en matériaux composites est entièrement activée, cette branche est très dépendante de la rigidité de l'enveloppe, elle-même directement proportionnelle au nombre de couches de composites collées.

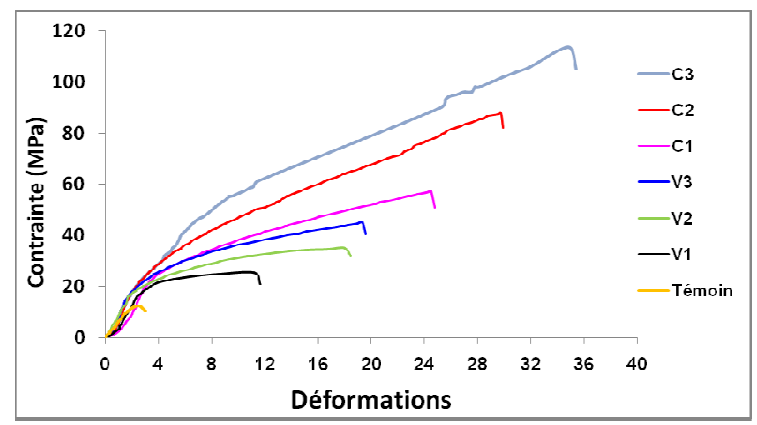

Fig. 4 Courbes contraintes-déformations des bétons testés

3.2 Variation de la résistance à la compression en fonction du nombre de couches 
D'après le tableau3, la charge ultime et les déformations axiales augmentent avec l'augmentation du nombre de couches.

D'autre part, les courbes présentées sur la Figure 5 montrent qu'une augmentation du nombre de couches du composite n'affecte pas la pente de la branche initiale de la relation contrainte-déformation, mais elle a un effet significatif sur la pente de la seconde branche.

Les corrélations obtenues à partir de la Figure 4, montrent que la variation de la résistance en compression en fonction du nombre de couches du composite est une fonction linéaire. Les fonctions de lissage ont des coefficients de variation $\mathrm{R}^{2}=0,998$. Ceci montre que la relation entre la résistance et le nombre de couches du composite $\mathrm{N}$ est correctement approchable par la fonction :

PRFG : $\mathrm{f}_{\mathrm{c}}=\mathrm{f}_{0}(32,33 \mathrm{~N}+20)$

PRFC $: \mathrm{f}_{\mathrm{c}}=\mathrm{f}_{0}(9,87 \mathrm{~N}+16)$

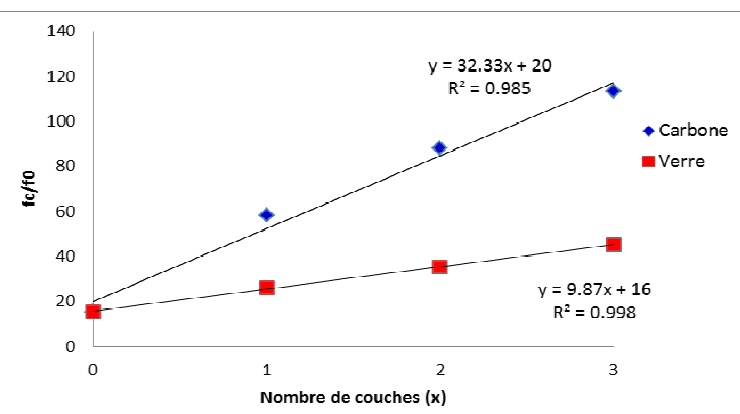

Fig. 5 Variation de la résistance à la compression en fonction du nombre de couches

\subsection{Mode de rupture du béton confiné}

La rupture des cylindres confinés avec la fibre de verre PRFG a été marquée par une rupture des fibres de verre dans le sens longitudinale du cylindre (Figure 6).

Dans tous les spécimens testés, la rupture a été fragile et d'une manière "brutale" due à l'énergie de déformation emmagasinée dans l'enveloppe composite au cours du chargement. Après la rupture, le béton fissuré suite à l'application de la contrainte de compression reste confiné à l'intérieur de l'enveloppe (Figure 7).

L'examen des spécimens après rupture, montre la présence d'une déchirure du composite le long d'une ligne verticale comme montré sur la Figure 5. Elle apparait pour les spécimens cylindriques dans les zones à faible résistance. Aucune délamination des tissus n'a été constatée et la forme conique du béton après rupture a été retrouvée.

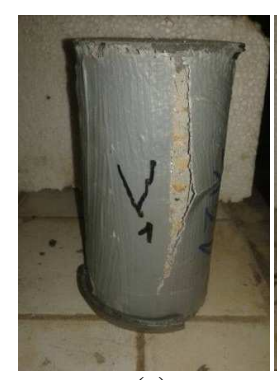

(a)

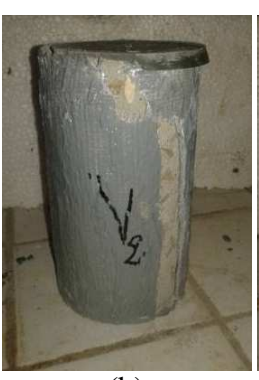

(b)

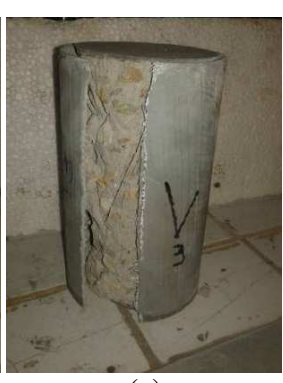

(c)
Fig. 6 Mode de rupture du béton confines par PRFC

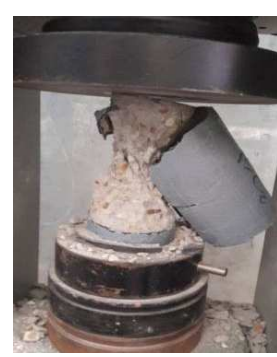

(a)

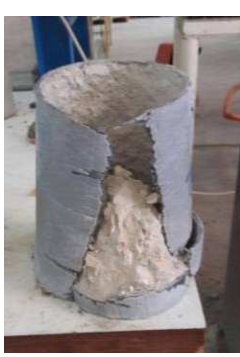

(b)

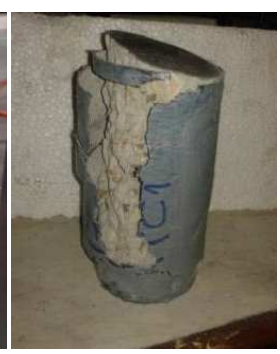

(c)
Fig. 7 Mode de rupture du béton confines par PRFG

\subsection{Résistances à la flexion}

Les résultats de l'essai de flexion 3 points sur les différents types des corps d'épreuve sont donnés dans le tableau 4.

Tableau 4. Résultats des essais sur les prismes en béton.

\begin{tabular}{|l|c|c|c|}
\hline $\begin{array}{c}\mathrm{N}^{\circ} \text { de couche } \\
\text { pour le béton }\end{array}$ & $\begin{array}{c}\text { Résistance } \\
\text { à la flexion } \\
(\mathrm{MPa})\end{array}$ & $\begin{array}{c}\text { Augmentations } \\
\text { des résistances } \\
(\%)\end{array}$ & $\begin{array}{c}\text { Allongement } \\
\text { A la rupture } \\
(\mathrm{mm})\end{array}$ \\
\hline $\mathrm{T}$ & 1,7 & - & 2,14 \\
\hline $\mathrm{V} 1$ & 6,2 & 265 & 1,93 \\
\hline $\mathrm{V} 2$ & 6,33 & 272 & 2,65 \\
\hline $\mathrm{V} 3$ & 7,01 & 312 & 2,97 \\
\hline $\mathrm{C} 1$ & 7,74 & 355 & 1,45 \\
\hline $\mathrm{C} 2$ & 8,14 & 379 & 1,67 \\
\hline $\mathrm{C} 3$ & 9,27 & 445 & 1,85 \\
\hline
\end{tabular}

Lors de l'essai de traction par flexion, on observe une augmentation considérable des forces de rupture pour chaque type de béton confiné (PRFC, PRFG) par rapport à celle du béton témoin. Dans tous les cas, on a réussi à augmenter la résistance et la ductilité.

Une augmentation du taux de confinement de 1, 2 et 3 couches de PRFG, à la flexion avec les spécimens non confinés et confinés avec le PRFC, conduit respectivement à une augmentation de la résistance à la traction par flexion des spécimens prismatiques confinés par PRFG de $265 \%$ pour une couche, $272 \%$ pour deux couches et $312 \%$ pour trois couches (Tableau 4).

Une augmentation du taux de confinement de 1,2 et 3 couches de PRFC, a la flexion avec les spécimens non confinés et confinés avec le PRFG, tel que montré sur la Figure 7 conduit respectivement à une augmentation de la résistance de traction par flexion des spécimens prismatiques confinés par PRFC de $355 \%, 379 \%$ et $445 \%$ tableau 4.

On retient de ces essais que la colle a un effet très limité sur la résistance à la traction du béton et donc à la flexion, tandis que le renforcement par la couche de PRFC en zone tendue augmente la résistance et la déformabilité du béton en flexion.

Le renforcement en PRFC en zone tendue, prolonge la résistance et la déformabilité du béton au delà de la première branche (béton témoin) par une seconde branche linéaire fragile de moindre rigidité. Cette perte de rigidité est due à la fissuration du béton.

Le tissu de verre confère une meilleure déformabilité qui pourra se traduire par une bonne ductilité. Le tissu de verre est 2 fois moins résistant que le carbone mais néanmoins deux fois plus ductile; il possède un pseudo- 
palier plastique de ductilité une fois la résistance ultime atteinte.

Cependant, le nombre de couche n'a pas une influence considérable sur l'amélioration de la résistance à la flexion (Figure 8).

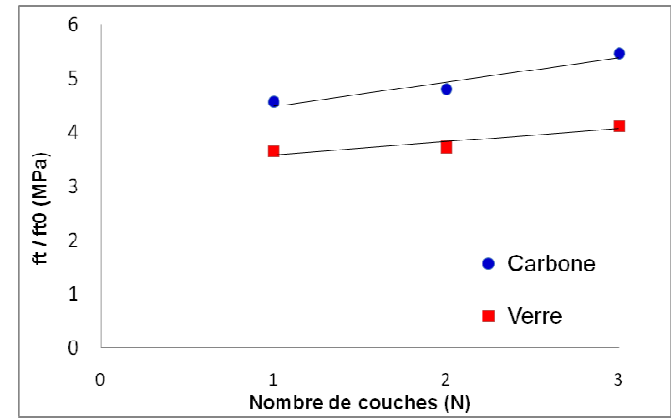

Fig. 8 Variation de la résistance à la traction en fonction du nombre de couches

\subsection{Mode de rupture du béton confiné}

L'élément renforcé par le tissu de carbone modifie le mode de rupture. On observe une fissure au niveau des appuis puis se propage au milieu, en suite son développement parallèlement à la charge appliquée au fur et à mesure que celle-ci augmente et le béton a été arraché dans les zones de rupture. Lors de l'essai de flexion trois point, on observe des fissures inclinée sur l'élément, au fur et à mesure que l'on augmente le taux de chargement (Figure 9).

La rupture des spécimens renforcés en PRFC est caractérisée par l'apparition de deux fissures quasiment symétriques inclinées d'un angle proche de $45^{\circ}$, la charge est diffusée par le biais de 2 bielles de compression qui soumettent le renforcement PRFC à une traction. Le composite n'a pas atteint sa limite ultime à la rupture. Celle-ci a eu lieu par traction du béton tendu suivi du délaminage de la couche de PRFC.

Concernant le tissu de verre, la rupture est obtenue par une rupture du tissu et la dégradation du béton est plus avancée que celle du béton témoin.

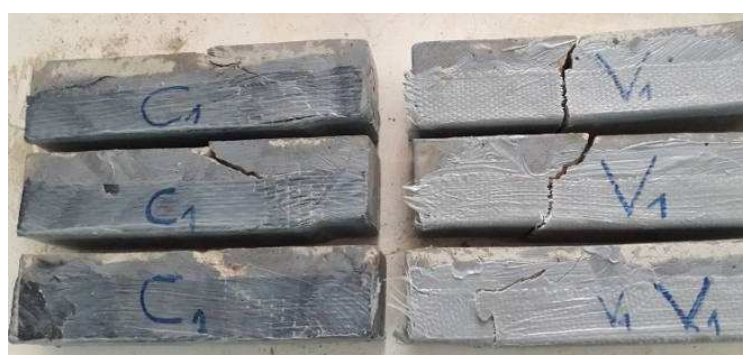

Fig. 9 Mode de rupture de béton de PRFC \& PRFG

\section{Conclusions}

A partir des résultats des essais, on peut tirer les conclusions suivantes :

- Résistance en compression: Le renfoncement par le tissu de verre a donné un gain de $63 \%$ pour $1 \mathrm{c}$ ouche et $120 \%$ pour 2 couches et $180 \%$ pour 3 couches. Le tissu de carbone à donné un gain de $260 \%$ pour 1 couche et $450 \%$ pour 2 couches et $607 \%$ pour 3 couches.

- Résistance à la flexion : L'augmentation a atteint $265 \%$ pour 1 couche et $272 \%$ pour 2 couches et $312 \%$ pour 3 couches pour le tissu de verre et $355 \%$ pour 1 couche, $379 \%$ pour 2 couches et $445 \%$ pour 3 couches pour le tissu de carbone.

- L'apport en rigidité du tissu composite aux éléments de structures est largement obtenu. Ceci permettra sans aucun doute, d'entreprendre par la suite des investigations plus poussées pour caractériser le matériau renforcé sous différentes sollicitations.

\section{Références}

1. A.Avril, "Application des méthodes de mesure de champs a la caractérisation mécanique de poutres en béton arme réparées par matériaux composites", Thèse de Doctorat, Ecole Nationale Supérieur des Mines de Saint-Étienne et de l'université Jean Monnet, 193 p, 2002.

2. S.O Alampalli, J. Connor, A.P. Yannotti. Fiber reinforced polymer composites for the superstructure of a short-span rural bridge”, Composites structures, Vol. 58, 2002, pp. 21-27

3. Y. H.Chai, , M. J. N. Priestley, et F. Seible "Seismic retrofit of circular bridge columns for enhanced flexural performance”, ACI Structural Journal, Vol. 88, No. 5, 1991, pp. 572-584 